\title{
Rare primary malignant peripheral nerve sheath tumor of the left testis: A case report
}

\author{
DONGDONG LIU ${ }^{1}$, YI MU ${ }^{1}$, PAN CHEN ${ }^{1}$, BANGWEI CHE ${ }^{1}$, ZHEMING LI ${ }^{1}$, \\ YILI ZHAO ${ }^{1}$, FA SUN $^{1}$ and KAIFA TANG ${ }^{1,2}$ \\ ${ }^{1}$ Department of Urology, Affiliated Hospital of Guizhou Medical University; \\ ${ }^{2}$ Institute of Medical Science of Guizhou Medical University, Guiyang, Guizhou 550004, P.R. China
}

Received September 26, 2020; Accepted April 9, 2021

DOI: $10.3892 / \mathrm{mco} .2021 .2306$

\begin{abstract}
Malignant peripheral nerve sheath tumor (MPNST) is a rare disease. The present study discusses the case of a 30 -year-old male patient who presented with complaints of pain in the left testis and groin over 1 month. Ultrasonography and computed tomography $(\mathrm{CT})$ imaging revealed the presence of a space-occupying lesion localized in the left testis. The mass was completely resected using an open surgical approach and was diagnosed as a primary MPNST of the left testis on postoperative histopathological examination. As this type of tumor is rare, there is currently no standard diagnostic or treatment method for MPNST. Pathological examination, enhanced CT imaging and immunohistochemical investigation are helpful for establishing the diagnosis and surgical resection is considered to be an effective treatment.
\end{abstract}

\section{Introduction}

Neurilemmoma, also referred to as Schwann cell tumor, originates from Schwann cells in the neuroectodermal lobe. The majority of neurilemmomas are benign tumors and malignant transformation is rarely observed (1). By contrast, malignant peripheral nerve sheath tumor (MPNST) is an aggressive neoplasm composed of spindled cells that may occur sporadically, and it often associated with radiation exposure or in the clinical context of neurofibromatosis type 1 (2). MPNST is rare and, to the best of our knowledge, it has not been reported outside of China. A case of intrascrotal schwannoma was first reported in Japan in 1991 and 6 more cases were recorded in the following years $(3,4)$. Only one case of testicular neurilemmoma was reported in China in 2003 (5). The presence of etiological factors, such as chronic low

Correspondence to: Professor Kaifa Tang, Department of Urology, Affiliated Hospital of Guizhou Medical University, 9 Beijing Road, Guiyang, Guizhou 550004, P.R. China

E-mail: tangkaifa@gmc.edu.cn

Key words: schwannoma, malignant, pathological examination, immunohistochemical, orchiectomy doses of ionizing radiation, may trigger carcinogenesis in the testicle (6).

The aim of the present study was to discuss a case of diagnosis and treatment of a rare case of testicular MPNST.

\section{Case report}

A 30-year-old man was admitted to the Department of Urology of the Affiliated Hospital of Guizhou Medical University (Guiyang, China) in August 2017 due to pain in the left testis and groin over 1 month. The patient reported no other symptoms. Physical examination identified a hard mass $(\sim 5.0 \times 5.0 \mathrm{~cm})$ in the left testis. The skin temperature of the scrotum was normal and scrotal light transmission test was negative. Urine culture and routine blood examination revealed no significant findings. Brightness-mode ultrasound examination revealed a mixed mass with unclear nature, sized $\sim 5.0 \times 5.0 \mathrm{~cm}$, in the left testis. Subsequently, computed tomography (CT) examination revealed a left testicular space-occupying mass and multiple enlarged retroperitoneal and bilateral peritesticular lymph nodes. Other laboratory tests (erythrocyte sedimentation rate and liver function) revealed no abnormal results.

After undergoing a thorough preoperative examination and obtaining consent from the patient and his immediate family, the patient underwent radical resection of the left testicular tumor (left testicular excision). After performing general anesthesia, the skin of the left scrotum was cut and the subcutaneous tissue and testis were gradually exposed. Macroscopically, the tumor was sized $\sim 5.0 \times 5.0 \mathrm{~cm}$, texture was hard and grayish white, the fascia of testis was closely adjoined to the skin. The left testis, epididymis and surrounding tissues were completely removed following vascular ligation and hemostasis. Histopathological examination of the removed tissue specimen confirmed the diagnosis of malignant peripheral neurilemmoma (Fig. 1). On hematoxylin and eosin staining, the tumor was composed of spindled tumor cells, whereas immunohistochemical examination revealed that the tumor cells were $\mathrm{CD} 57^{+}$and $\mathrm{S}-100^{+}$, as well as identifying the presence of giant tumor cells. The patient recovered well following the operation and was discharged 7 days after operation. After discharge, the patient was lost to follow-up. 

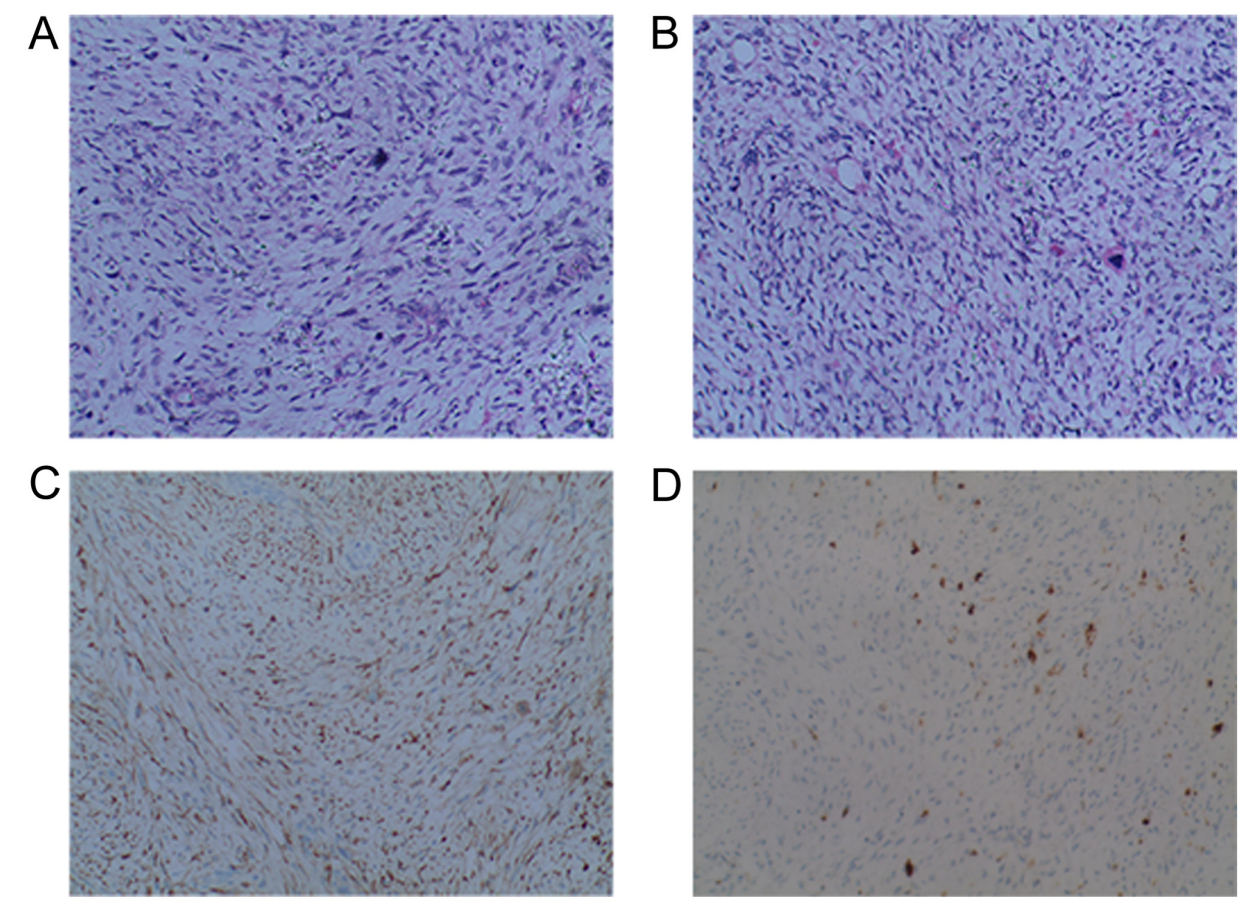

Figure 1. Histopathological examination of the resected specimen. (A and B) Hematoxylin and eosin staining of the tumor. Immunohistochemical staining of the tumor revealed that the tumor cells were positive for the expression of (C) CD57 and (D) S-100. Magnification, x100.

\section{Discussion}

Schwannoma commonly originates from tissues with abundant nerve supply, such as the stomach, ears and parotid glands (7-9). The most common age of patients with neurilemmomas is $30-50$ years. Most cases are benign, and malignant transformation is rarely observed. The presence of additional etiological factors, such as chronic low doses of ionizing radiation, may initiate the process of carcinogenesis in the testicle (6). Tumor growth is slow in the early stages. However, with increased tumor volume, compression symptoms of adjacent organs may develop. In the urinary system, the primary symptoms are pain and urinary tract irritation. Microscopically, schwannoma is characterized by a constantly changing Antoni A region (dense cellular area) and Antoni B region (hypocellular area). In the highly differentiated Antoni A area, the nuclei may be seen arranged in a in palisade pattern, and mitotic figures are occasionally observed (10).

Primary MPNST is a rare and poorly understood entity. The diagnosis of MPNST relies on clinical manifestation and pathological results, whereas histopathological diagnosis predominantly involves light microscopy (11). Imaging examination is helpful in terms of localization and qualitative diagnosis of the tumor. Brightness-mode ultrasound examination revealed a nodular hypoechoic mass with a clear boundary. On CT scan, the tumor density was slightly lower compared with that of muscle or presented as mixed density. On MRI, benign schwannomas are lightly hyperintense relative to muscle on T1-weighted images and have a target appearance on T2-weighted images with a peripheral hyperintense rim and central hypointensity. Intense contrast enhancement is seen in most schwannoma cases (12). The typical target appearance may not be present in MPNST (12). However, the lack of MRI data may be a limitation to this study.
The primary treatment for schwannomas is surgery (13). Particularly for MPNSTs, in order to prevent tumor recurrence, the main tumor and its surrounding tissue should be completely removed to the greatest extent possible (13). The role of chemotherapy in MPNSTs has not been clearly determined. A series of chemotherapy drugs have been used in patients with MPNST and have shown some effectiveness (14). It has been reported that there is a certain association between testicular cancer and sarcoidosis, further contributing to the uncertainty surrounding the treatment of MPNSTs (15). With the rapid development of biological characterization and molecular biology techniques, novel targeted drugs and gene therapy may improve the treatment of this disease.

At present, literature remains sparse on the prognostic factors and ideal treatment methods to control MPNSTs. While surgical excision with negative margins and radiation therapy seems to be the current mainstay, the prognosis remains poor (16). In the present case, the tumor location was superficial, resection was complete, therefore, the prognosis was good. However, long-term follow-up of MPNSTs is necessary due to the possibility of recurrence.

\section{Acknowledgements}

Not applicable.

\section{Funding}

No funding was received.

\section{Availability of data and materials}

All data generated or analyzed during this study are included in this published article. 


\section{Authors' contributions}

KT conceptualized and designed the study. DL, PC, BC and YM performed the experiments. ZL, FS and YZ analyzed the data. DL wrote the manuscript. YM and KT reviewed and edited the manuscript. All authors confirm the authenticity of all the raw data. All authors read and approved the final manuscript.

\section{Ethics approval and consent to participate}

The present study was approved by the Ethics Committee of the Affiliated Hospital of Guizhou Medical University (approval no. 2013023). The patient provided written informed consent.

\section{Patient consent for publication}

The patient agreed to the collection and publication of the present case study.

\section{Competing interests}

The authors declare that they have no competing interests.

\section{References}

1. Davis DD and Kane SM: Neurilemmoma. StatPearls Publishing, Treasure Island, FL, 2021.

2. Mishra B, Madhusudhan KS, Kilambi R, Das P, Pal S and Srivastava DN: Malignant schwannoma of the esophagus: A rare case report. Korean J Thorac Cardiovasc Surg 49: 63-66, 2016.

3. Shimizu H, Tsuchiya A and Kusama H: A case of intrascrotal neurilemmoma. Hinyokika Kiyo 37: 303-304, 1991 (In Japanese).
4. Matsui F, Kobori Y, Takashima H, Amano T and Takemae K: A case of intrascrotal schwannoma. Hinyokika Kiyo 48: 749-751, 2002 (In Japanese).

5. Jiang R, Chen JH, Chen M and Li QM: Male genital schwannoma, review of 5 cases. Asian J Androl 5: 251-254, 2003.

6. Bazalytska SV, Persidsky Y and Romanenko AM: Molecular mechanisms of initiation of carcinogenesis in the testis. Exp Oncol 41: 224-234, 2019.

7. $\mathrm{Pu} \mathrm{C}$ and Zhang K: Gastric schwannoma: A case report and literature review. J Int Med Res 48: 300060520957828, 2020.

8. Kim KS, Lee H, Choi JH, Hwang JH and Lee SY: Schwannoma of the posterior branch of the great auricular nerve. Arch Craniofac Surg 21: 368-371, 2020.

9. Chowdhary S, Thangavel S, Ganesan S and Alexander A: Extratemporal intraparotid facial nerve schwannoma. BMJ Case Rep 14: e239407, 2021.

10. Donnelly MJ, al-Sader MH and Blayney AW: Benign nasal schwannoma. J Laryngol Otol 106: 1011-1015, 1992.

11. James AW, Shurell E, Singh A, Dry SM and Eilber FC: Malignant peripheral nerve sheath tumor. Surg Oncol Clin N Am 25: 789-802, 2016.

12. Anil G and Tan TY: Imaging characteristics of schwannoma of the cervical sympathetic chain: A review of 12 cases. AJNR Am J Neuroradiol 31: 1408-1412, 2010.

13. Halliday J, Rutherford SA, McCabe MG and Evans DG: An update on the diagnosis and treatment of vestibular schwannoma. Expert Rev Neurother 18: 29-39, 2018.

14. Bradford D and Kim A: Current treatment options for malignant peripheral nerve sheath tumors. Curr Treat Options Oncol 16: 328, 2015.

15. Bassoulet J, du Chatelard P, L'Her P, Natali F, Morel C, Cosnard G, Merrer J, Guillemot MC and Timbal Y: Cancer of the testis, sarcoidosis and neurinoma. Ann Urol (Paris) 24: 219-323, 1990 (In French).

16. Mullins BT and Hackman T: Malignant peripheral nerve sheath tumors of the head and neck: A case series and literature review. Case Rep Otolaryngol 2014: 368920, 2014.

(i) (3) This work is licensed under a Creative Commons Attribution-NonCommercial-NoDerivatives 4.0 International (CC BY-NC-ND 4.0) License. 